\title{
The propagation of Elastic Waves in Granular Solid Hydrodynamics
}

\author{
Michael Mayer and Mario Liu \\ Institut für Theoretische Physik, Universität Tübingen, 72076 Tübingen, Germany, EU
}

( $\Omega$ Dated: March 23, 2022)

\begin{abstract}
The anisotropic, stress-dependent velocity of elastic waves in glass beads - as observed by Y. Khidas and X. Jia, see [Phys. Rev. E, 81:021303, Feb. 2010] - is shown to be well accounted for by "granular solid hydrodynamics," a broad-range macroscopic theory of granular behavior. As the theory makes no reference to fabric anisotropy, the influence of which on sound is in doubt.
\end{abstract}

PACS numbers: 91.60.Lj, 81.40.Jj

\section{INTRODUCTION}

The anisotropic spectrum of elastic waves in granular media is studied in [1]. Wave velocity was measured under varying stress, in glass bead samples that have undergone two different preparations: Rain deposition that consists of pouring the glass beads into the acoustic cell through a mesh; and de-compaction, implying the gentle motion of a horizontal grid through the bead pack, from the bottom to the top, after the deposition. As the velocity was seen to depend on the preparation, and because it is known from numerical simulations that the rain deposition creates an anisotropic distribution of the contact angles, with two preferred directions oriented roughly at $30^{\circ}$ from the vertical, this was taken as evidence of the influence of fabric anisotropy.

However, also reported was the fact that the preparations lead to different densities. So a crucial question is whether the density alone suffices to explain the observed difference in the velocity. Only if this is not the case, would the experimental evidence imply that fabric anisotropy exerts independent influence, that it is in fact a state variable - in addition to, and independent from, others such as the stress, density, velocity, and the granular temperature. Generally speaking, of course, fabric anisotropy could well behave like many other local or microscopic variables, is different in different circumstances but, being a dependent quantity, would be given if the complete set of state variables is.

Fig. 1 demonstrates that the velocity difference can indeed be explained by the density difference. Although there is some residual discrepancy between theory and observation, many possible reasons remain, making the case for fabric anisotropy less than cogent: The calculation was carried out under simplifying assumptions, and the experiment contains deviation from ideal conditions. The employed theory, GSH (for granular solid hydrodynamics) 2], contains only two elastic coefficients, leaving the energy independent of the third strain invariant, see section [II] On the experimental side, possibly neither the density nor the static stress is uniform, as assumed. The latter is mainly a result of the elliptical geometry, and the inhomogeneity in density should especially be dependent on preparation.

Having adjusted the parameters such that the density difference from the two preparations suffices to explain the velocity difference in the compressional waves, Khidas and Jia find that the discrepancy in the shear wave velocities remains, and depends on the preparation, see their Fig. 8. Accepting this as an evidence for the independent influence of fabric anisotropy, however, presumes complete confidence in the density dependence of

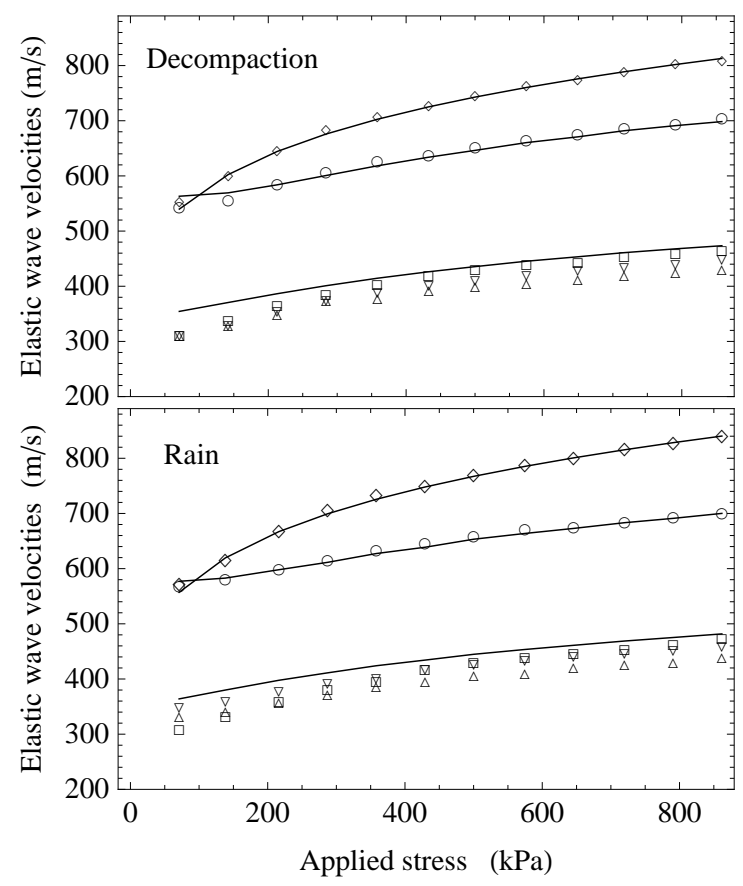

FIG. 1: Symbols represent measured wave velocities versus the applied stress $\sigma_{33}$, as reported by Khidas and Jia, for two different preparations, decompaction and rain, corresponding respectively to the packing ratio of $\phi=0.606$ and $\phi=0.643$. (The geometry is triaxial, the preferred direction along the vertical is denoted as 3.) More specifically, rhombuses are vertical compressional waves $c_{v}$; circles horizontal ones $c_{h}$. The remaining three are shear waves, with varying direction and polarization, see [1]. The lines are theoretical results, obtained by employing GSH with density-dependent elastic coefficients, but excluding any possible independent influences from preparations. In the employed approximation of two elastic coefficients, assuming uniaxiality, GSH yields the same velocity for all three shear waves - a justified approximation, as their difference is clearly small. 
EMT, the theory employed in [1] to account for the wave velocities. GSH sports a more realistic density dependence [2 5], and the same discrepancy is small. In addition, the neglected elastic coefficients associated with the third strain invariant should also depend on the density, further reducing the discrepancy.

We derive the elastic modes from GSH in section II, and apply the results to the experimental circumstances of [1] in section [II]

\section{ELASTIC MODES IN THE GSH THEORY}

GSH theory is a hydrodynamic theory meant to account for a wide spectrum of granular behavior, see [2 5] for derivation and explanation. Its variables consist of the density $\rho$, momentum $\rho v_{i}$, the elastic strain $u_{i j}$, and two entropies, the true and the granular one, $s, s_{g}$. To consider elastic waves, we only need the equations

$$
\begin{aligned}
\partial_{t}\left(\rho v_{i}\right)+\nabla_{j}\left(\sigma_{i j}+\rho v_{i} v_{j}\right) & =0, \\
\partial_{t} u_{i j}-(1-\alpha) v_{i j}+\frac{u_{i j}^{0}}{\tau}+\frac{u_{l l} \delta_{i j}}{\tau_{1}} & =0,
\end{aligned}
$$

with the stress given as $\sigma_{i j}=(1-\alpha) \pi_{i j}-\zeta_{g} v_{l l} \delta_{i j}-\eta_{g} v_{i j}^{0}$, $\pi_{i j} \equiv-\partial w / \partial u_{i j}$, and $w$ denoting the elastic energy. The coefficients $\alpha$ and $\tau^{-1}, \tau_{1}^{-1}$ account respectively for the typical granular phenomena of stress softening and strain relaxation that give rise to plasticity. These coefficients and the viscosities $\eta_{g}, \zeta_{g}$ are all functions of the granular temperature and vanish for $T_{g} \rightarrow 0$. For small wave amplitudes, the granular temperature $T_{g}$ is also small, making all these effects negligible, and granular media essentially elastic. This is the limit we shall consider here, while postponing the account of wave dissipation from viscosity and plasticity to a forthcoming paper. Taking $\alpha, \tau^{-1}, \tau_{1}^{-1}, \eta_{g}, \zeta_{g}=0$, and $u_{i j}=\frac{1}{2}\left(\partial_{i} U_{j}+\partial_{j} U_{i}\right)$, we have

$$
\rho \ddot{U}_{i}+M_{i j k l} \nabla_{j k} U_{l}=0,
$$

a wave equation for the displacements $U_{i}$, with the stressdependent stiffness tensor [3], $M_{i j k l}=\partial^{2} w / \partial u_{i j} \partial u_{k l}$. We take the granular elastic energy to be

$$
w=\mathcal{B} \sqrt{\Delta}\left(2 \Delta^{2} / 5+u_{s}^{2} / \xi\right)
$$

with $\Delta \equiv-u_{l l}, u_{s}^{2} \equiv u_{i j}^{0} u_{i j}^{0}$, and the superscript ${ }^{0}$ denoting the traceless part of any tensor, eg. $u_{i j}^{0} \equiv$ $u_{i j}+\delta_{i j} \Delta / 3$. The two elastic coefficients are $\mathcal{B}$ and $\xi$, where $\xi$ is a constant, and $\mathcal{B}$ density dependent,

$$
\mathcal{B}=\mathcal{B}_{0}\left(\frac{\phi-\phi_{l p}^{*}}{\phi_{c p}-\phi}\right)^{\frac{3}{20}}
$$

where $\phi \equiv \rho / \rho_{G}$ is the packing ratio, with $\phi_{c p}, \phi_{l p}$ respectively the ratio of closest and loosest packing, and $\phi_{l p}^{*}$ given by $\phi_{l p}=\left(11 \phi_{c p}+9 \phi_{l p}^{*}\right) / 20$.
Inverting the stress-strain relation $\pi_{i j}=\pi_{i j}\left(u_{k l}\right)$, taking $P \equiv \pi_{l l} / 3$ and $\pi_{s}^{2} \equiv \pi_{i j}^{0} \pi_{i j}^{0}$, we express the elastic strain as

$$
\begin{array}{r}
2 L=1+\sqrt{1-\xi \pi_{s}^{2} /\left(2 P^{2}\right)} \\
\Delta=(L P / \mathcal{B})^{\frac{2}{3}}, \quad u_{s}=\pi_{s} /(2 \mu), \\
u_{i j}^{0}=-\frac{\pi_{i j}^{0}}{2 \mu}, \quad \mu=\frac{\mathcal{B}}{\xi}\left(\frac{L P}{\mathcal{B}}\right)^{1 / 3} .
\end{array}
$$

For uniform stress and strain, in the principal axes of both the stress and strain [see the first of Eqs (6)], $\pi_{i j}=\pi_{(i)} \delta_{i j}$, the stiffness tensor has the simple form

$$
\begin{aligned}
M_{i j k l} & =\frac{\mathcal{B}}{\xi}\left(\frac{L P}{\mathcal{B}}\right)^{1 / 3}\left(a_{(i k)} \delta_{i j} \delta_{k l}-\delta_{i k} \delta_{j l}-\delta_{i l} \delta_{j k}\right) \\
a_{i k} & \equiv\left(\frac{\xi \pi_{s}}{4 L P}\right)^{2}+\frac{4-9 \xi}{6}-\frac{\xi\left(\pi_{i}^{0}+\pi_{k}^{0}\right)}{2 L P}
\end{aligned}
$$

where there is no summation over bracketed subscripts. So, for instant

$$
M_{1122}=\frac{\mathcal{B}}{\xi}\left(\frac{L P}{\mathcal{B}}\right)^{\frac{1}{3}}\left[\left(\frac{\xi \pi_{s}}{4 L P}\right)^{2}+\frac{4-9 \xi}{6}+\frac{\xi\left(\pi_{1}^{0}+\pi_{2}^{0}\right)}{2 L P}\right] .
$$

A plane wave ansatz $\sim \exp (\omega t+\vec{k} \vec{x})$ to eq. (1) results in the eigenvalue problem $\left(K_{i l}-\lambda \delta_{i l}\right) U_{l}=0$, where

$$
\begin{array}{r}
K_{i l} \equiv\left(\beta_{(i)}+\beta_{(l)}\right) n_{i} n_{l} / 2-\delta_{i l}, \\
c^{2} \equiv \omega^{2} / k^{2}=-\lambda \mathcal{B}(L P / \mathcal{B})^{\frac{1}{3}} /(\rho \xi), \\
\beta_{i} \equiv 1-Q-\frac{\xi \pi_{i}^{0}}{L P}, \quad Q \equiv \frac{3 \xi}{2}+\frac{4}{3}-\left(\frac{\xi \pi_{s}}{4 L P}\right)^{2},
\end{array}
$$

and $n_{i}=k_{i} / k$. Eigenvalues $\lambda$ yield the velocity of propagation $c$, and the corresponding eigenvectors $U$ the polarization. The general solution is given by the eigenvalues $\lambda_{1}=-1$ and $\lambda_{2,3}=\frac{1}{2}(\eta \mp \sqrt{\zeta}-2)$, where $\eta \equiv \sum_{i=1}^{3} \beta_{i} n_{i}^{2}$ and $\zeta \equiv \sum_{i=1}^{3} \beta_{i}^{2} n_{i}^{2}$.

As long as $\beta_{1} \neq \beta_{2} \neq \beta_{3}$, and the wave vector is not along one of the principal directions, the associated displacements are

$$
\begin{aligned}
U_{1}= & \left(\begin{array}{l}
n_{2} n_{3}\left(\beta_{2}-\beta_{3}\right) \\
n_{3} n_{1}\left(\beta_{3}-\beta_{1}\right) \\
n_{1} n_{2}\left(\beta_{1}-\beta_{2}\right)
\end{array}\right), \\
U_{2,3}= & \left(\begin{array}{l}
n_{1}\left(\zeta \mp \sqrt{\zeta}\left(\beta_{1}+\beta_{1}\right)+\beta_{1} \beta_{1}\right) \\
n_{2}\left(\zeta \mp \sqrt{\zeta}\left(\beta_{1}+\beta_{2}\right)+\beta_{1} \beta_{2}\right) \\
n_{3}\left(\zeta \mp \sqrt{\zeta}\left(\beta_{1}+\beta_{3}\right)+\beta_{1} \beta_{3}\right)
\end{array}\right) .
\end{aligned}
$$

In the case of propagation along principal directions the tensor $K$ is diagonal from the very beginning, and we have, eg., for $n_{1}=1, \lambda_{1}=\beta_{1}-1$ and $\lambda_{2,3}=-1$ with $U_{1}=(1,0,0), U_{2}=(0,1,0)$ and $U_{3}=(0,0,1)$. Thus, plane waves traveling along the principal directions of the static stress field are either pure longitudinal or transversal modes. 
For an isotropic background stress we have $\pi_{i}^{0}=0$, and $\beta_{1}=\beta_{2}=\beta_{3}$, and the compressional wave velocity shows the typical $P^{\frac{1}{6}}$ Hertz-scaling,

$$
c_{p}=\sqrt{\left(\frac{3}{2} \xi+\frac{4}{3}\right) \frac{1}{\xi \phi} \frac{\mathcal{B}}{\rho_{G}}} \sqrt[6]{\frac{P}{\mathcal{B}}} .
$$

For a uniaxial state, with 3 as the preferred direction, we have $\beta_{1}=\beta_{2}$ and $\lambda_{1}=-1, \lambda_{2,3}=\frac{1}{2}(\eta \mp \sqrt{\zeta}-2)$, with $\eta \equiv\left(\beta_{1}-\beta_{3}\right) n_{1}^{2}+\beta_{3}, \zeta \equiv\left(\beta_{1}^{2}-\beta_{3}^{2}\right) n_{1}^{2}+\beta_{3}^{2}$. Depending on $n_{1}$ alone, $\lambda$ (and therefore also the velocities $c$ ) possesses rotational symmetry. The compressional wave velocity along 3 , or $n_{1}=0$, is given by

$$
c_{v}=\sqrt{\left(Q+\frac{\pi_{3}^{0}}{L P}\right) \frac{1}{\xi \phi} \frac{\mathcal{B}}{\rho_{G}}} \sqrt[6]{\frac{L P}{\mathcal{B}}} .
$$

The lateral compressional velocity, $n_{1}=1$, is

$$
c_{h}=\sqrt{\left(Q+\frac{\pi_{1}^{0}}{L P}\right) \frac{1}{\xi \phi} \frac{\mathcal{B}}{\rho_{G}}} \sqrt[6]{\frac{L P}{\mathcal{B}}} .
$$

Only for vanishing shear, $\pi_{i}^{0}, \pi_{s} \rightarrow 0, L \rightarrow 1$, do both formulas reduce to $\mathrm{Eq}(9)$. Otherwise, the $\sqrt[6]{P}$ - Hertzscaling is not valid. The two shear wave modes for each of the two directions all have the eigenvalue, $\lambda=-1$, so

$$
c_{s}=\sqrt{\frac{1}{\xi \phi} \frac{\mathcal{B}}{\rho_{G}}} \sqrt[6]{\frac{L P}{\mathcal{B}}} .
$$

\section{ANISOTROPY OF GRANULAR SOUND}

These results are now applied to analyze the data of [1]. From Eqs (10[11), we first calculate, for the uniaxial case, the ratio of vertical and horizontal compressional velocities,

$$
\left(\frac{c_{v}}{c_{h}}\right)^{2}=\frac{L P Q+\pi_{3}^{0}}{L P Q+\pi_{1}^{0}}
$$

which clearly does not depend on $\mathcal{B}$, only on $\xi$, determined as $\xi=3 / 4$ from the measured velocities of both preparations, rain and decompaction. Clearly, $\xi$ does not depend much on the density, as assumed. Then, taking $\phi_{l p}=0.555$ and $\phi_{c p}=0.664$, any data point will fix $\mathcal{B}_{0}$ as $5.1 \mathrm{GPa}$. The result is Fig 1, In fact, data points from compressional waves were used to fix $\xi$ and $\mathcal{B}_{0}$, so the curves of shear wave velocities are free of fit parameters.

The degenerate velocity for shear waves is a consequence of the simple form given for the energy in Eq (2). Generally speaking, there are three strain invariants: $\Delta \equiv-\operatorname{Tr} \hat{u}, u_{s}^{2} \equiv \operatorname{Tr} \hat{u}^{2}-\frac{1}{3}(\operatorname{Tr} \hat{u})^{2}$, and $u_{I I I} \equiv(\operatorname{Tr} \hat{u})^{3}-$ $3 \operatorname{Tr} \hat{u} \operatorname{Tr} \hat{u}^{2}+2 \operatorname{Tr} \hat{u}^{3}$, of which only the first two enter the expression of Eq (2). Including the third invariant, we did find a discrimination of shear wave velocities under static shear. [Note that although $u_{I I I}$ is of third order, its energy contribution, say $\sim u_{I I I} / \sqrt{\Delta}$, may well be of the same order as the terms already in Eq (2).] We are at present looking for a suitable extension of Eq (2), taking into account in addition its effect on the Coulomb yield.

\section{SUMMARY}

We have shown that GSH, employing only two independent elastic coefficients, $\mathcal{B}_{0}=5.1 \mathrm{GPa}, \xi=3 / 4$, is capable of reproducing the experimental date of [1] fairly well. Various possible reasons for the remaining disagreement are given and weighted, all rather more tangible than fabric anisotropy. Moreover, the density dependence of $\mathcal{B}$ and the density independence of $\xi$ is further validated.
[1] Y. Khidas and X. Jia. Anisotropic nonlinear elasticity in a spherical-bead pack: Influence of the fabric anisotropy. Phys. Rev. E, 81:021303, Feb. 2010.

[2] M. Liu and Y. Jiang. Granular solid hydrodynamics. Granular Matter, 11(3):139-156, May 2009.

[3] Y. Jiang and M. Liu. Incremental stress-strain relation from granular elasticity: Comparison to experiments. Physical Review E (Statistical, Nonlinear, and Soft Matter Physics), 77(2):021306, 2008.
[4] K. Bräuer, M. Pfitzner, D. O. Krimer, M. Mayer, Y. Jiang, and M. Liu. Granular elasticity: Stress distributions in silos and under point loads. Physical Review E (Statistical, Nonlinear, and Soft Matter Physics), 74(6):061311, 2006.

[5] D. O. Krimer, M. Pfitzner, K. Bräuer, Y. Jiang, and M. Liu. Granular elasticity: General considerations and the stress dip in sand piles. Physical Review E (Statistical, Nonlinear, and Soft Matter Physics), 74(6):061310, 2006. 